\title{
A WAVELENGTH RECOGNIZING SWITCHING ARCHITECTURE FOR OMEGA INTERCONNECTION NETWORKS
}

\author{
Andrea Borella, Giovanni Cancellieri, Pietro Prosperi \\ Dipartimento di Elettronica ed Automatica, Università di Ancona Via Brecce Bianche, 60131 \\ Ancona, Italy
}

\begin{abstract}
Wavelength Recognizing Switches (WRS) allow the self-routing of optical packets in a flexible way, by maintaining the header processing in the optical domain. Therefore these devices can be adopted within a multistage interconnection network to build a modular switching architecture. This paper proposes and analyzes an interconnection topology based on an Omega network graph, where the stages are composed by WRS modules, focusing some solutions to perform add and drop of packets, internal routing procedures and control wavelengths management. The system suitability in implementing efficiently the optical burst switching paradigm is discussed.
\end{abstract}

Key words: Optical switching, WDM, Omega networks, MP $\lambda$ S, cross-talk

\section{STATE-OF-THE-ART OF OPTICAL SWITCHING}

At present, in the nodes of an OTN (Optical Transport Network) the switching function is realized in a circuit switching mode, using traditional electronic fabrics. This solution implies a double conversion of the physical nature of the propagating signals at the input and output stages. Despite the theoretical possibility of adopting devices able to switch in the optical domain, such components are not fully defined and still far from their industrial implementation.

In any case, among the possible architectures, conceived in order to operate as optical switching matrices, the WXC (Wavelength Cross 
Connect) exploits the potentialities offered by WDM. They can be classified as follows:

- pure WXC;

- WXC with space switching fabrics;

- WXC with space switching fabrics and wavelength converters.

A non-fully accessible network characterizes pure WXCs, whereas those equipped with space matrices (if dimensioned properly) are fully accessible and non-blocking. Finally, the insertion of lambda converters allows wavelength rearrangement within the switching node [1].

The physical implementation of WDM (de)multiplexers is based on passive devices called AWGM (Arrayed Waveguide Grating Multidemultiplexer). Alternatively, a $2 \times 2$ basic element can be built using a couple of optical circulators and a diffraction grating filter. On the other hand, the space switching fabric is usually based on a MEM (MicromachineElectro-Mechanical) structure, composed by arrays of electrically driven micro-mirrors. Finally, the lambda conversion can be obtained by four-wave mixing, cross-phase modulation, or adopting a Mach Zenhder interferometer with a phase modulator.

On the side of optical packet switching technique, the main problem is represented by the unsatisfactory state of the art as concerns optical buffering. In fact, at present, delay lines are the only solution available as photonic memory. It is evident that, in practice, only few packets could be stored in them; in addition such solution is not compatible with a compact integration of the different devices composing the future switching boards.

The utilization of delay lines implies fixed length packets, whose duration would be a compromise between opposite requirements. In fact the header of very short packets, crossing the node too fast, could not be processed within the time limits available to perform routing in the node [2]. On the contrary, long packets are heavy to store in the small optical memories.

As an example, having a signal propagating at a speed equal to $2 \cdot 10^{8} \mathrm{~m} / \mathrm{s}$ and a bit rate of $10 \mathrm{Gbit} / \mathrm{s}$, a delay lines able to store 500 bits is 10 meters long. The space consumed to built bigger memories increase accordingly. This fact makes the research on different buffering techniques advisable [3], [4], [5].

However, the hypothetical optical buffers, when available, can be placed either at the input or at the output ports of the switching section [6]. In both cases, wavelength converters have to be included in order to simplify the contention resolution problem, occurring when more than one packet on the same wavelength should reach a given output.

With respect to the discussed techniques, a more advanced switching solution seems to be represented by the wavelength recognizing method, 
where particular wavelengths are reserved and explicitly used for routing processing at the optical layer. Such method is focused in sections 3 and 4 . As it will be shown, it is suitable either for circuit switching or packet switching, even though it is particularly devoted to optical burst switching.

The paper is organized as follows. In Section 2. the optical burst switching paradigm is presented and analyzed. Section 3 . focuses the principles of the wavelength recognizing switching, whereas the adoption of this technique within multistage networks is discussed in Section 4. Finally, Section 5 . concludes the paper.

\section{OPTICAL BURST SWITCHING}

An integrated architecture, where WDM techniques are not only confined in the transport of optical signals, in a point-to-point or circuit switched context, is a very important goal.

In fact, the increased amount of requested bandwidth, especially due to the evolution of Internet services, asks for a complete exploitation of the optical potentialities. In addition, the enormous increment of traffic due to the expansion of such services seems to make the migration towards packet switching mode preferable. Thus, to develop an optical Internet, $\mathbb{P}$ layer has to drive the WDM one, obtaining an effective integration between such two levels.

In this sense the switching node represents the bottleneck. Among the possible switching techniques for optical networks, neither wavelength routing (that is a form of circuit switching) nor optical packet switching (for the reasons explained before) seem to offer immediate perspective. Obviously, the former is not coherent with the support of a ubiquitous, decentralized IP network (essentially because of the few available wavelengths and low bandwidth utilization), while the latter cannot be fully developed at present. In fact, as the analysis reported in the previous section indicates, all-optical networks must avoid photonic buffering as much as possible. Furthermore, at the bit rates available on fibers, the header processing becomes impossible at packet level. Moreover, since optical RAM are not available at present, many of the QoS (Quality of Service) schemes conceived for a packet switching context are not applicable at the WDM layer.

On the contrary, if a way of aggregating IP packets into burst of suitable length is defined, some of the above mentioned problems could be overcome. This is the approach of the so called OBS (Optical burst switching) [7]. OBS is a sort of compromise between packet and circuit switching techniques. In practice, an aggregation of packets composes a 
burst, which can be handled as a packet itself, but within time limits sufficient for the routing process. After that, having obtained a good integration among higher layers and physical layer protocols, the redundancy due to functions repeated at different levels can be greatly reduced, so incrementing the dimension of the payload size with respect to the header one. In this way, in an interconnected high speed network [8], routing processes and traffic engineering can be performed electronically, but information can cross the mass-of-glass without interruptions.

The considered integration can be obtained through different approaches such as MPLS (MultiProtocol Label Switching) [9], its extension MPAS (MultiProtocol lambda Switching) [9] or LOBS (Label OBS) [7], similar to MP $\lambda S$ but proposed for wavelength-routed networks.

\section{THE WRS SCHEME}

The considered technique is based on a basic module, called WRS (Wavelength Recognizing Switch) [10]. It is a $1 \times 2$ photonic gate, exploiting the non-collinear four-wave-mixing effect within a broad-area SOA (Semiconductor Optical Amplifier). Its fundamental role concerns the routing of an incoming WDM signal towards the selected output, by interpreting the presence or absence of specific routing wavelengths inside the spectrum of the same signal. The routing process is fully executed without requiring any optoelectric conversion. The WRS, which has a fourth port used to receive control wavelengths, set to drive the internal switching, handles particular incoming signals composed by two optical bands, according to the principle illustrated in the sequel.

The first band contains a sequence of routing wavelengths, which anticipate the second wavelength sequence, carrying the payload. The entire signal (signaling and data) is routed towards a given port if one of the routing wavelengths is almost coincident with a control wavelength generated locally. Otherwise, the signal crosses the alternative port.

A simple but interesting scheme is drawn in Figure 1, where the operation illustrated previously is reduced to the management of only 2 wavelengths incoming at port $\mathrm{I}, \lambda_{\mathrm{r}}$ for signaling and $\lambda_{\mathrm{s}}$ for data. Moreover, port $\mathrm{C}$ is the input for the control wavelength $\lambda_{\mathrm{c}}$.

As said before, if $\lambda_{c}$ matches $\lambda_{\mathrm{r}}$ (within the recognizing bandwidth), $\lambda_{\mathrm{s}}$ is routed towards port $T$, otherwise it does not deflect its direct path, reaching port $P$.

The WRS utilizes a deflection mechanism triggered by the interference, inside the SOA, among the routing and control wavelengths, that generates a running grating of index and gain [11]. A small shift $\delta \lambda$ can affect signal at 
output $T$, depending of an eventual difference between $\lambda_{c}$ and $\lambda_{r}$. In addition to its all-optical operation, the medium response time (less than a nanosecond) allows WRS to switch at a frequency compatible with the bit rate characterizing an optical backbone.

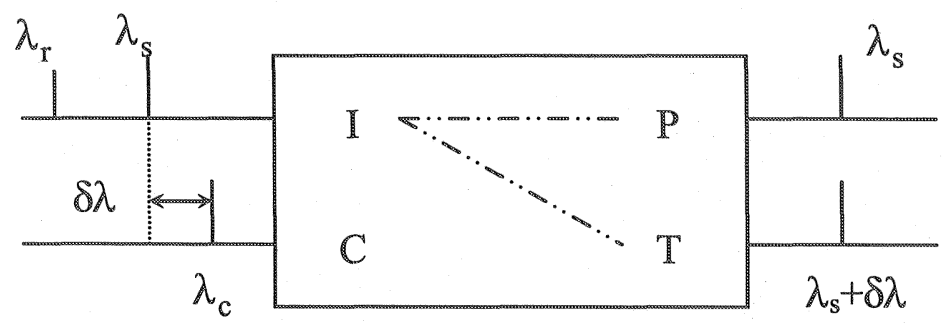

Figure 1. The WRS operation; port P: direct signal, port T: signal deflected.

The efficiency of the switching process, defined as the ratio between the signal power when routed at port $\mathrm{T}$ and that of the same incoming signal at port I, reaches its maximum value when $\lambda_{\mathrm{c}}$ and $\lambda_{\mathrm{r}}$ are tuned perfectly, whereas it decreases as $\delta \lambda$ gets higher. From that definition, the recognizing bandwidth corresponds to an efficiency reduction equal to $3 \mathrm{~dB}$, with respect to its maximum value. Experimentally, that bandwidth has been measured at $0.57 \mathrm{GHz}$, with a switching bandwidth (the wavelength range switched by WRS) of $42 \mathrm{~nm}$ [11].

As concerns the crosstalk, in the WRS it is equal to the ratio between the signal power at port $\mathrm{T}$, being $\lambda_{\mathrm{c}}$ and $\lambda_{\mathrm{r}}$ tuned perfectly, and the power of the signal at the same port when the control wavelength is not injected. In this case, experimental measurements estimate a value of $-28.8 \mathrm{~dB}$.

Insertion and extraction of wavelengths travelling along a WDM or DWDM (Dense WDM) fiber backbone can be realized through WRS-based OADM (Optical Add-Drop Multiplexer) devices, similar to that reported in Figure 2. In that scheme, where there are three different source/destination (S/D) local ports, the two feedback delay lines are utilized to avoid packet collisions during the insertion process only, while the routing wavelength are inserted within the dashed block. Finally, $\mathrm{S}$ is an optical filter that discriminates the wavelengths devoted to local information exchange, with respect to those destined to be injected in the fiber. Analogously, the S-array operates in the same way, but working on the backbone signal.

Now, let us focus the attention on the internal structure of the space switching matrix, composed by WRS elements, similar to that used in the OTN core. 
The structure of a basic $2 \times 2$ WRS switching element (SE) is reported in Figure 3 a), where it is assumed that the routing wavelengths are injected in the fibers at the previous hop, performing a sort of remote set-up of the SE. In this way, however, taking into account that such element is part of a multistage interconnection network, the adjacent nodes should be informed about the internal structure of the switching fabric. Since this approach does not seem to be realistic, a solution where nodes exchange routing information via MP $\lambda S$ is preferable. In turn, each node will make its routing choices on its own internal interconnection graph. Therefore, assuming that routing information is processed at the higher IP layer, and transmitted to the WDM one via network binding and interfaces, at the optical domain the proper control wavelengths will be generated and presented at the inputs of the WRS stages. Consequently, we can adopt the scheme of Figure $3 \mathrm{~b}$ ), which works as explained in the sequel.

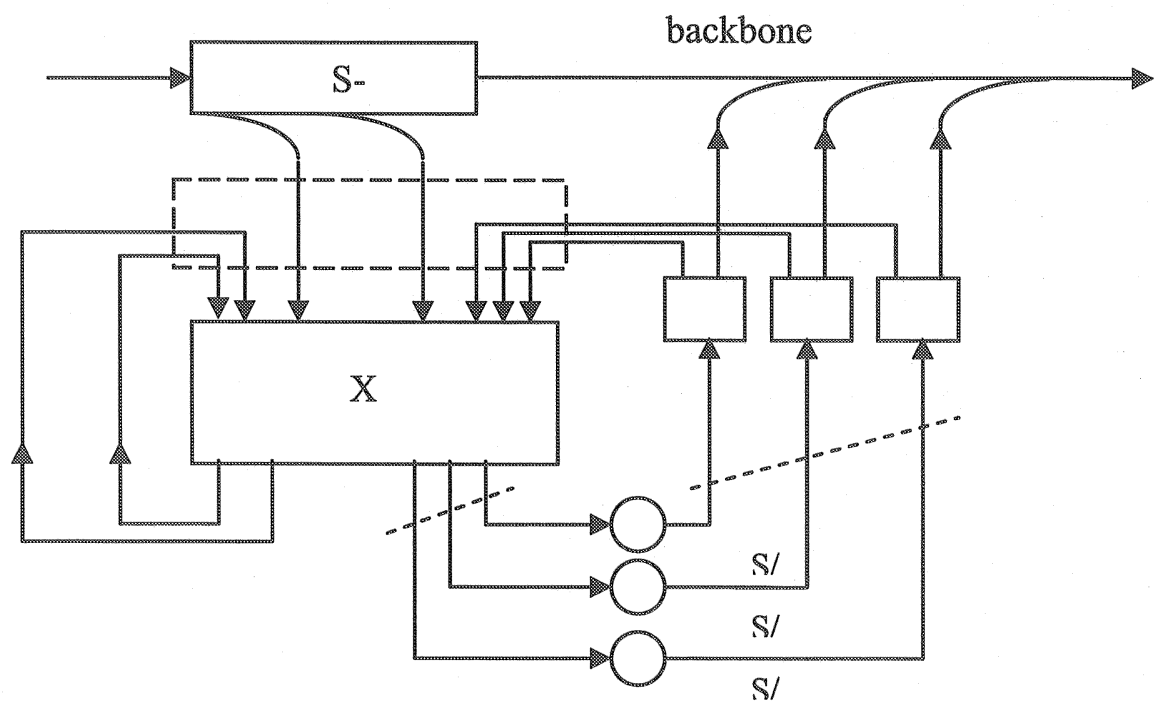

Figure 2. AODM based on a WRS switch.

According to the mechanism of wavelength matching previously explained, cross- and bar-state configurations can be selected, by injecting or not the control wavelength respectively. In particular, if $\lambda_{c}$ is switched off we have the following connections: $\mathbb{N}_{0}$-OUT 0 and $\mathbb{N}_{1}$-OUT 1 (bar-state). On the contrary, when $\lambda_{c}$ is on, we have $\mathbb{N}_{0}$-OUT 1 and $\mathbb{N}_{1}$-OUT (cross- $_{0}$ state). It is necessary to stress that there is not any problem of wavelength shifting, since the same optical source drives either the routing or the control wavelength on the two input ports of each WRS, which are obviously 
perfectly tuned. Thus, the condition of zero detuning also implies the maximization of the switching process efficiency. At the same time, in absence of $\lambda_{c}$, there is not any coupling effect with the routing wavelength and this fact minimizes the crosstalk among outputs.

Operating in a OBS context, the incoming bursts are long enough to allow the operation of the routing layer, which defines the proper configuration of the switching fabric. The latter is imposed by driving the gates which provide the control wavelengths. In principle, the proposed procedure is not far from the concept of self-digit-controlled networks.

By combining multiple $2 \times 2$ WRS SEs, large OMIN (Optical Multistage Interconnections Networks) can be built, as shown in the next Section.

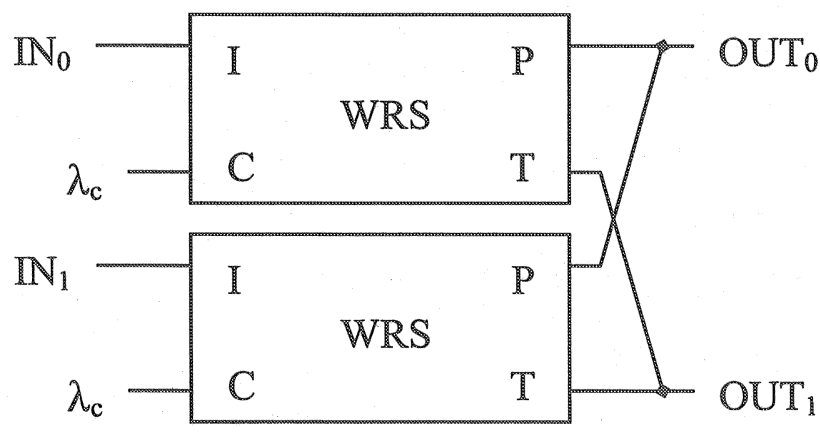

a)

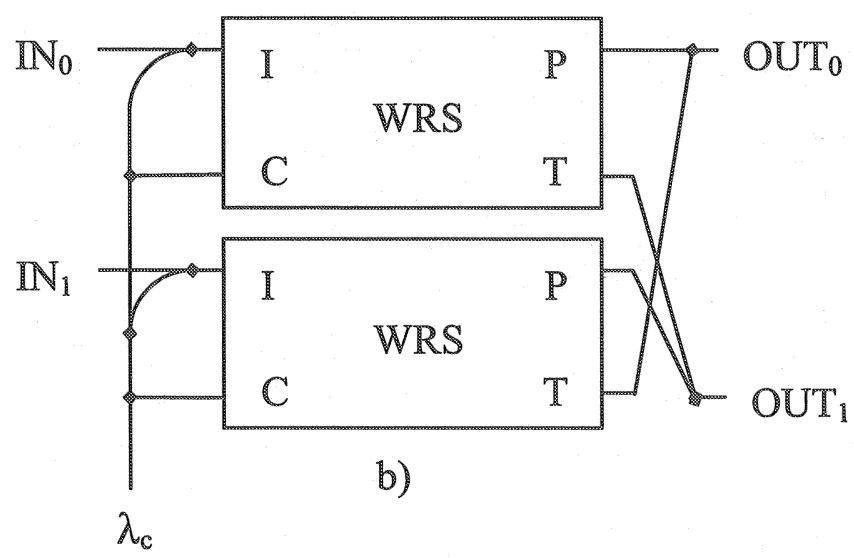

Figure 3. The 2x2 WRS switching module: a) remote b) local WRS set-up. 


\section{A WRS OMEGA INTERCONNECTION NETWORK}

Among the various topology proposed as structure of OMINs, we have focused the attention on Omega networks [12]. Then, we have developed a possible Omega architecture using WRS SEs.

A $\mathrm{N} \times \mathrm{N}$ Omega network, with $\mathrm{N}=2^{\mathrm{n}}$, is composed by $\mathrm{n}$ stages (columns), each of them including a number of $2 \times 2$ SEs equal to $N / 2$. From the previous description of such elements in the WRS technology, it follows that $N \cdot \log _{2} \mathrm{~N}$ WRS components are necessary in this topology. The internal patterns follows different rules which depends on the considered stage. In fact the inter-stage connections adopt the shuffle-exchange scheme [13]. Similarly, the input ports of the network are also shuffled before reaching the first stage. On the contrary, the output ports of the last stage are directly connected the network outputs.

An example of a $4 \times 4$ topology is reported in Figure 4, where a previous wavelength demultiplexing is assumed. Since the goal is the analysis of the WRS utilization in optical interconnection networks, we have depicted this scheme that is characterized by a very small input dimension. So, even though the omega pattern is in this case very simple, we can observe that only one control wavelength is necessary to perform internal routing in the whole switching fabric. 


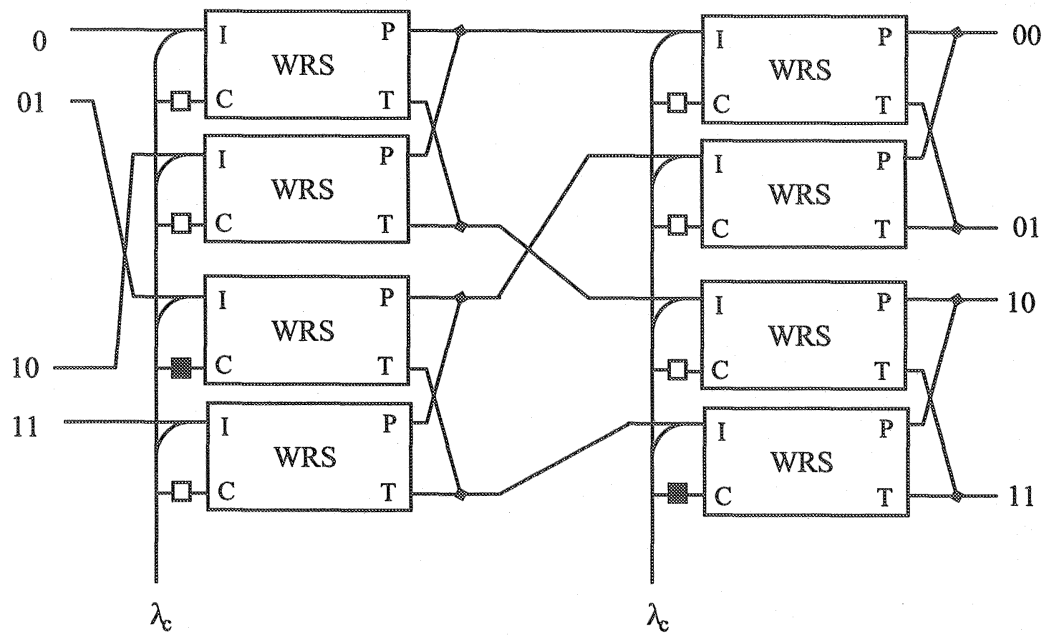

Figure 4. A 4x4 Omega interconnection network based on WRS modules.

However, it is worth noting that, with respect to the scheme of Figure 3 b), same gates are placed at the C port of each WRS. They can present or not the $\lambda_{c}$ at that port, according their ON or OFF state respectively. This is a way of showing how the switch can be controlled and how the routing choices can be transferred at the optical level, to connect every input with its proper output. Furthermore, the connection at the output of the WRS SEs have been modified accordingly, to drawn a structure equivalent to that of Figure $3 \mathrm{~b}$ ). Finally, the $\mathrm{N}$ inputs and outputs are numbered in a binary format.

In practice, once having received at the $\mathbb{P}$ level the signaling information, the gates are set in order to obtain the desired permutation. At the i-th stage, with $0 \leq \mathrm{i} \leq \mathrm{n}-1$, along the considered input-output path, the i-th bit of the output address has to be used to drive the corresponding gate, where bits 0 and 1 correspond to the $\mathrm{OFF}$ and $\mathrm{ON}$ state respectively. In the example of Figure 4, to switch the signal at input 01 towards the 11 output, the first and the second gate (drawn in black color) have to be set ON (since both the bits of the destination address equals 1 ).

Following the proposed approach, the considered network can be scaled to increase its dimension. However, the scalability is affected by the amount of crosstalk interference suffered by the signals travelling across the fabric. As said before, the crosstalk effect is minimized by according the routing and the control wavelengths (as in this case, where they coincide). Nevertheless, a further contribution to crosstalk is due to the simultaneous presence of two signals at the two port I of a WRS SE. See for example the 
same example of Figure 4, considering that inputs 00 and 10 ask for connection to outputs 00 and 11 respectively. In this case, even though the two paths are different, at the output $\mathrm{T}$ of the first WRS at stage 0 a certain interfering power tapped from the signal outgoing from port $\mathrm{P}$ (directed to output 00 ) affects the signal at the exit of port $T$ of the second WRS in the same SE (directed to output 11).

The crosstalk problem can be solved by avoiding that two signals have access to the same WRS SE. This can be done, for example, using the RTDM (Reconfiguration with Time Division Multiplexing) [14] approach, which distributes the input-output connections among different crosstalk free (CF) permutations, mapping the interconnection network in a TDM way. In other words, colliding paths are allocated in different time slots through suitable algorithms, so that no more than one signal has a simultaneous access to a given WRS SE. In this sense, we are developing possible routing protocols, able to define CF patterns within WRS Omega networks.

For the being, it is worth noting that the proposed scheme is not properly a hybrid OMIN [15], where switch control and routing decision are carried out electronically. In fact the WRS modules are managed at the OTN layer, and no electric signals are used inside their fabric. According to the MP $\lambda S$ stack, in this architecture the data plane of the optical cross connect uses an interconnection network to provision a lightpath from an input port to an output port [9], leaving to the label switched routers, at the IP layer, the task of discovering, distributing and maintaining the relevant state information about the MP $\lambda S$ network.

\section{FUTURE PERSPECTIVES}

This paper has discussed the adoption of the WRS switching element as a module of OMINs, operating in a MP $\lambda S$ context, assuming optical burst switching as a way to match the necessity of data packetization in the present state-of the art of photonic technologies. As evidenced, interesting perspective are connected to this solution, which seems to approach many of the problem which block the full deployment of all-optical networks.

Nevertheless, other important aspects need further analysis. In particular, our present research activity is focused on the optical crosstalk effect, which is one of the main concern as regards QoS in OTNs. We are working to control it, by developing specific algorithms devoted to identify suitable crosstalk-free (CF) permutations to be set in the Omega interconnection network. In particular, the goal is the recovery of partial CF permutation, by a progressive erasing of conflicting paths within a non-CF configuration. Adopting the philosophy of deflection routing [13], those paths are 
rearranged and directed towards different outputs (with respect to those identified by the routing tables) to obtain a new CF setup. Once again, assuming an effective interaction among the protocols of the MP $\lambda S$ stack, a prioritized management of the traffic can be developed, in order to force the deflection of lowest service classes first. This will be the subject of the next paper.

\section{REFERENCES}

[1] Chen Y.K., Lee C.C., Fiber Bragg grating-based large nonblocking multiwavelength cross-connects, Lightwave Technology, 1998, 16:1746-1756.

[2] Borella A., Chiaraluce F., Proposal of a packet switch based on purely random routing, International Journal of Communication Systems, 1996, 9:145-150.

[3] Yamanaka Y., Numai T., Kasahara K., Kubota K., Optical fiber loop memory using vertical to surface transmission electro-photonic device, Journal of Lightwave Technology, $1993,11: 2140-2144$.

[4] Huffaker D.L., Lee W.D., Deppe D.G., Lei C., Rogers T.J., Campbell J.C., Streetman B.G., Optical memory using a vertical-cavity surface-emitting laser, IEEE Transactions Photonics Technology Letters, 1991, 3:1064-1066.

[5] Nakajima K., Kan H., Mizushima Y., Power-speed product of an optical flip-flop memory with optical feedback, IEEE Journal of Solid-State Circuits, 1991, 26:75-76.

[6] Zhong W.D., Tucker R.S., Wavelength routing-based photonic packet switching systems, Journal of Lightwave Technology, 1998, 16:1737-1745.

[7] Qiao C., Labeled optical burst switching for IP-over-WDM integration, IEEE Communication Magazine, 2000, 38:104-114.

[8] Borella A., Cancellieri G., Chiaraluce F., Design techniques of two-layer architectures for WDM optical networks, International Journal of Communications, 2001, 14:171-188.

[9] S.Seetharaman, "IP over optical networks: a summary of issues", Internet Draft, October 2001, http://search.ietf.org//internet-drafts/drafts-osu-ipo-pmls-issues-02.txt.

[10] Hoanca B. et al., All-optical routing using wavelength recognizing switches, Journal of Lightwave Technology, 1998, 16:2243-2253.

[11] Zhu D.X. et al., A novel all-optical switch: the wavelength recognizing switch, $\mathbb{E E E E}$ Photonics Technology Letters, 1997, 9:1110-1112.

[12] Shen X., Yang F., Pan Y., Equivalent permutation capabilities between time division optical omega Networks and Non-optical Extra-Stage Omega Networks, IEEE/ACM Transactions on Networking, 2001, 9:518-524.

[13] Borella, Cancellieri, Chiaraluce, Wavelength division multiple access optical networks, Norwood (USA): Artech House, 1998.

[14] Qiao C., Melhem R., Chiarulli D., Levitan S., A time domain approach for avoiding crosstalk in optical blocking Multistage Interconnection Networks, IEEE Journal of Lightwave Technology, 1994, 12:1854-1862.

[15] Pan Y., Qiao C., Yang Y., Optical multistage interconnection networks: new challenges and approaches, IEEE Communications Magazine, 1999, 37:50-56. 